\title{
Identification of thyroxine-binding globulin as a candidate plasma marker of chronic obstructive pulmonary disease
}

This article was published in the following Dove Press journal:

International Journal of COPD

25 May 2017

Number of times this article has been viewed

\section{Wenqi Diao' \\ Ning Shen' \\ Yipeng Du' \\ Xiaoyan Sun' \\ Beibei Liu' \\ Ming $\mathrm{Xu}^{2,3}$ \\ Bei $\mathrm{He}^{1}$}

'Department of Respiratory Medicine, ${ }^{2}$ Department of Cardiology, Institute of Vascular Medicine, Peking University Third Hospital,

${ }^{3}$ Key Laboratory of Molecular Cardiovascular Sciences, Ministry of Education, Beijing, People's Republic of China
Correspondence: Bei He

Department of Respiratory Medicine, Peking University Third Hospital, 49 North Garden Road, Haidian District, Beijing, I0019I, People's Republic of China

Tel +861082265210

Fax $+86 \quad 1082086810$

Email puh3_hb@bjmu.edu.cn
Abstract: Biomarkers for the management of chronic obstructive pulmonary disease (COPD) are limited. The aim of this study was to explore new plasma biomarkers in patients with COPD. Thyroxine-binding globulin (THBG) was initially identified by proteomics in a discovery panel and subsequently verified by enzyme-linked immunosorbent assay in another verification panel with a 1-year follow-up. THBG levels were elevated in patients with COPD $(9.2 \pm 2.3 \mu \mathrm{g} / \mathrm{mL})$ compared to those of the controls $(6.6 \pm 2.0 \mu \mathrm{g} / \mathrm{mL})$. Receiver operating characteristic curves suggested that THBG was able to slightly differentiate between patients with COPD and controls (area under the curve [AUC]: 0.814) and performed better if combined with fibrinogen (AUC: 0.858). THBG was more capable of distinguishing Global Initiative for Obstructive Lung Disease stages I-III and IV (AUC: 0.851) compared with fibrinogen (AUC 0.582). THBG levels were negatively associated with predicted percentage forced expiratory volume in $1 \mathrm{~s}$ and positively related to predicted percentage residual volume, RV/percentage total lung capacity, and percentage low-attenuation area. COPD patients with higher baseline THBG levels had a greater risk of acute exacerbation (AE) than those with lower THBG levels $(P=0.014$, by Kaplan-Meier curve; hazard ratio: 4.229 , by Cox proportional hazards model). In summary, THBG is a potential plasma biomarker of COPD and can assist in the management of stable stage and AEs in COPD patients.

Keywords: thyroxine-binding globulin, biomarker, chronic obstructive pulmonary disease, proteomics

\section{Introduction}

Chronic obstructive pulmonary disease (COPD) is a complicated disease characterized by the progressive decline of lung function with or without emphysema, leading to considerable morbidity and mortality worldwide. ${ }^{1}$ Biomarkers, such as proteins, microRNAs, and metabolites, play an important role in the diagnosis and management of various complex diseases. ${ }^{2-5}$ However, biomarkers for the diagnosis and management of COPD are very limited. ${ }^{6,7}$ Moreover, these markers, such as fibrinogen and C-reactive protein (CRP), appeared to reflect systemic inflammation in patients with COPD. ${ }^{8-12}$ Most researchers in the respiratory field would prefer to identify a biomarker involved in the pathological mechanism of COPD. Thus, researchers are trying to identify new biomarkers to replace or complement these traditional markers.

Recently, proteomics has been widely applied in the identification of biomarkers in various diseases due to the high-throughput and unbiased characteristics of the technique. ${ }^{13-17}$ Likewise, the technique has also been used in biomarker studies of COPD ${ }^{18-20}$ Several markers identified by proteomic studies have provided new insights 
into the pathogenesis and management of COPD. ${ }^{18,19,21,22}$ Samples of these proteomic studies were obtained from sputum, ${ }^{21}$ exhaled breath condensate (EBC), ${ }^{23}$ or bronchoalveolar lavage fluid (BALF). ${ }^{24}$ However, sputum is not a suitable sample for proteomic studies because highly charged mucins can easily cause the separation of sputum proteins in the proteomics process. ${ }^{25}$ The application of EBC in proteomics is also limited due to salivary contamination and poor protein abundance. ${ }^{26}$ BALF is an ideal material for the identification of specific biomarkers related to the pathogenesis of COPD, but protein dilution and high risk restrict its clinical application. ${ }^{27}$ Plasma is an appropriate sample because it is easily obtained and contains abundant human proteins.

To our knowledge, plasma biomarker studies using proteomics in stable COPD patients are scarce. ${ }^{18}$ Hence, this study sought to identify new plasma biomarkers in COPD via the isobaric tags for relative and absolute quantification (iTRAQ)-labeling proteomic technique. To verify these candidate biomarkers identified by proteomics, their plasma concentrations were measured in another larger cohort with a 1-year follow-up by enzyme-linked immunosorbent assay (ELISA). Furthermore, we also explored the relationship between biomarkers and clinical characteristics of COPD.

\section{Methods}

\section{Study design}

A discovery panel of 8 male subjects $(n=4$, smokers without COPD; $n=4$, smokers with COPD) was recruited to identify the biomarker candidates in November 2014. Another verification panel of 86 male subjects $(n=33$, smokers without COPD; $n=53$, smokers with COPD) was used to verify and explore the clinical value of biomarkers. The subjects were enrolled and followed up from December 2014 to February 2016. All recruited subjects were from the Peking University Third Hospital, China. The study protocol was approved by the Medical Science Research Ethics Committee of Peking University Third Hospital (2014160), and all studies were carried out in strict accordance with the protocol. Written informed consent was obtained from each subject. The flow diagram is presented in Figure 1.

The inclusion criteria of COPD patients were as follows: 1) males aged 50-70 years; 2) diagnosed with a stable stage of COPD, according to the Global Initiative for Obstructive Lung Disease (GOLD) guidelines ${ }^{1}$; and 3 ) a smoking history of at least 10 pack-years and a smoking cessation duration of $>5$ years. The exclusion criteria of selected patients were as follows: 1) diagnosed with unstable cardiovascular diseases, significant hepatic dysfunction, or mental or renal incompetence; 2) diagnosed with asthma, diffuse panbronchiolitis, cystic fibrosis, active pulmonary tuberculosis, or pneumonia within 2 months; or 3 ) prescribed immunosuppressive medications, such as those for acquired immunodeficiency syndrome (AIDS). The COPD patients were divided into 4 subgroups according to the baseline lung function: GOLD I (predicted percentage forced expiratory volume in $1 \mathrm{~s}$ $\left[\mathrm{FEV}_{1} \%\right.$ pred $\left.] \geq 80 \%\right)$, GOLD II $\left(50 \% \leq \mathrm{FEV}_{1} \%\right.$ pred $\left.<80 \%\right)$, GOLD III $\left(30 \% \leq \mathrm{FEV}_{1} \%\right.$ pred $\left.<50 \%\right)$, and GOLD IV $\left(\mathrm{FEV}_{1} \% \text { pred }<80 \%\right)^{1}$. In addition, male smokers without COPD were included as a control group.

Age, gender, body mass index (BMI), smoking history, smoking cessation duration, complications, and 1-year acute exacerbation (AE) information were collected. Lung function was measured with a ventilator (SensorMedics, Yorba Linda, CA, USA).

The diagnosis of AE was based on GOLD guidelines. ${ }^{1}$ No subjects were hospitalized due to AEs in the study. The severity of emphysema was assessed according to the extent of low-attenuation area (LAA) measured by thorax computed tomography (CT). LAA\%, defined as the percentage of the area of $<-950$ Hounsfield units (HU), was calculated with AW version 4.5 software (GE Healthcare, Fairfield, CT, USA). ${ }^{28}$

\section{Sample collection}

Plasma samples from subjects were centrifuged for $30 \mathrm{~min}$ at room temperature. Supernatants of these blood samples were aliquoted into sterile Eppendorf tubes and subsequently stored at $-80^{\circ} \mathrm{C}$.

\section{ITRAQ-labeling proteomic analysis}

The highly abundant plasma proteins were discarded with ProteoMinerTM kits (Bio-Rad Laboratories, Hercules, CA, USA). The extracted proteins from plasma were digested via Trypsin Gold (Promega, Madison, WI, USA) for $16 \mathrm{~h}$ at $37^{\circ} \mathrm{C}$. The plasma samples of subjects were labeled with the 8-plex iTRAQ reagent (Applied Biosystems, Carlsbad, CA, USA). These samples were pooled after being incubated for $2 \mathrm{~h}$ at room temperature. The peptides were separated by strong cation exchange (SCX) chromatography through an LC-20AB HPLC pump system (Shimadzu, Kyoto, Japan) and then processed by liquid chromatography coupled with tandem mass spectrometry (LC-MS/MS) with a Q Exactive ${ }^{\mathrm{TM}}$ mass spectrometer (Thermo Fisher Scientific, San Jose, CA, USA). Proteins were identified against the UniProt_human database via a mascot search engine version 2.3.02 (Matrix Science, London, UK). 


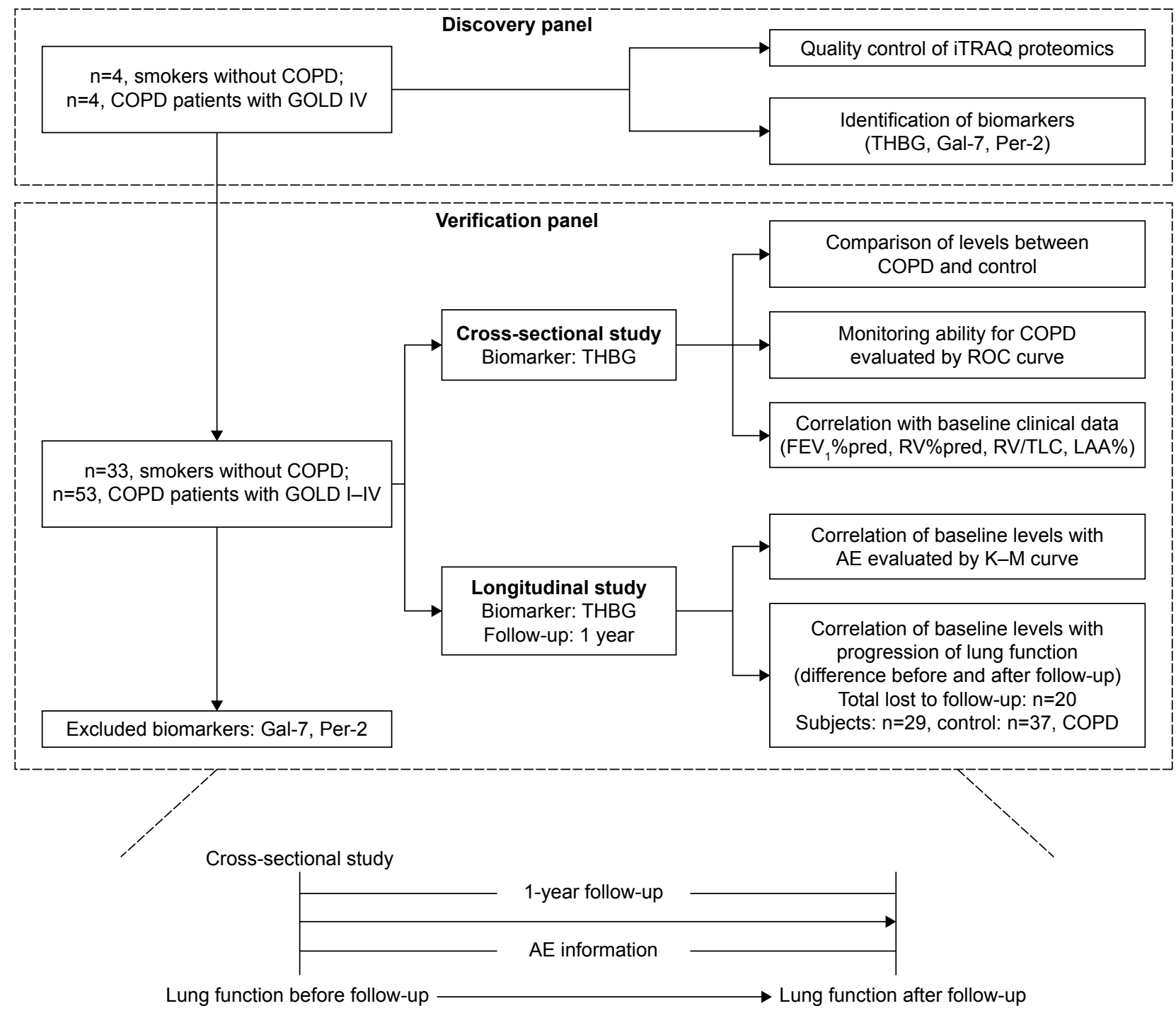

Figure I Flow diagram of the study.

Abbreviations: AE, acute exacerbation; COPD, chronic obstructive pulmonary disease; FEV,\%pred, predicted value of forced expiratory volume in I s; Gal-7, galectin-7; GOLD, Global Initiative for Obstructive Lung Disease; iTRAQ, isobaric tags for relative and absolute quantification; K-M curve, Kaplan-Meier curve; LAA\%, percentage of low-attenuation area; Per-2, peroxirodoxin-2; ROC, receiver operating characteristic; RV\%pred, predicted value of residual volume; THBG, thyroxine-binding globulin; RV/TLC, the ratio of the residual volume and the total lung capacity.

\section{Immunoassay verification}

Thyroxine-binding globulin (THBG) (R\&D Systems, Minneapolis, MN, USA), peroxiredoxin-2 (R\&D Systems), and galectin-7 (Invitrogen, Grand Island, NY, USA) were detected using ELISA kits according to the manufacturers' protocols. Plasma fibrinogen concentration was measured by the Clauss method. ${ }^{8}$ Total triiodothyronine $\left(\mathrm{TT}_{3}\right)$, total thyroxine $\left(\mathrm{TT}_{4}\right)$, free $\mathrm{T}_{3}\left(\mathrm{fT}_{3}\right)$, free $\mathrm{T}_{4}\left(\mathrm{fT}_{4}\right)$, and thyroidstimulating hormone (TSH) were determined with an automated chemiluminescence immunoassay (Roche Elecsys, Basel, Switzerland) ${ }^{29}$

\section{Statistical analysis}

Categorical variables were assessed with the chi-square test. Differences in continuous variables between controls and patients with COPD were compared using Student's $t$-tests. Discrepancies in continuous variables among controls and patients at GOLD stages I-IV were evaluated by analysis of variance (ANOVA) with a post hoc test (least significant difference [LSD] method). Analyses of covariance and multivariate linear regression, correcting for confounders, were used to identify differences in THBG between groups.

The association of THBG with continuous variables was determined by the Pearson coefficient, and the association with ranked variables was analyzed by Spearman's coefficient. Partial correlation analyses and multivariate linear regression, correcting for confounders, were used to detect the relationship between THBG and variables.

Receiver operating characteristic (ROC) curves were used to detect the partitive ability of THBG for different subgroups 
of COPD, including the corresponding area under the curve (AUC), the cutoff value, sensitivity, and specificity.

The COPD patients were divided into low-level and highlevel groups based on the median concentration of baseline THBG. A Kaplan-Meier curve was used to compare the occurrence time of AEs between low- and high-level THBG groups. A Cox proportional regression model, correcting for other risk factors, was used to detect the hazard ratio (HR) of THBG levels for AEs.

Statistical analyses were conducted through GraphPad Prism 5.0 (GraphPad, San Diego, CA, USA), MedCalc ${ }^{\circledR} 15.1$ (MedCalc, Ostend, Belgium), and SPSS 19 (SPSS, Chicago, IL, USA).

\section{Results}

\section{Identification of THBG by proteomics in the discovery panel}

The discovery panel, which included 8 male smokers with or without COPD, was used to identify biomarker candidates. Details are presented in Table S1.
The quality of the iTRAQ-labeling proteomics is assessed in Figure S1. Thirteen proteins were upregulated and 28 were downregulated in COPD patients compared to those in controls. The expression profile is listed in Table S2. Because of the limited availability of kits, only THBG, galectin-7, and peroxiredoxin-2 were verified by ELISA. Figure S2 shows that the relative intensities of the 3 proteins quantified by iTRAQ were similar to their relative concentrations measured by ELISA.

THBG was initially identified based on the representative MS/MS spectrum (-GWVDLFVPK-) with 7 typical $b$-ions and $5 y$-ions, as shown in the upper panel of Figure 2. Levels of THBG in COPD patients were higher than in smokers without COPD, as presented in the lower panel of Figure 2.

\section{Validation of candidates by ELISA in the verification panel}

The verification panel, which included 86 male smokers with or without COPD, was used to verify these candidates via ELISA. Detailed information about the subjects is shown in Table 1.

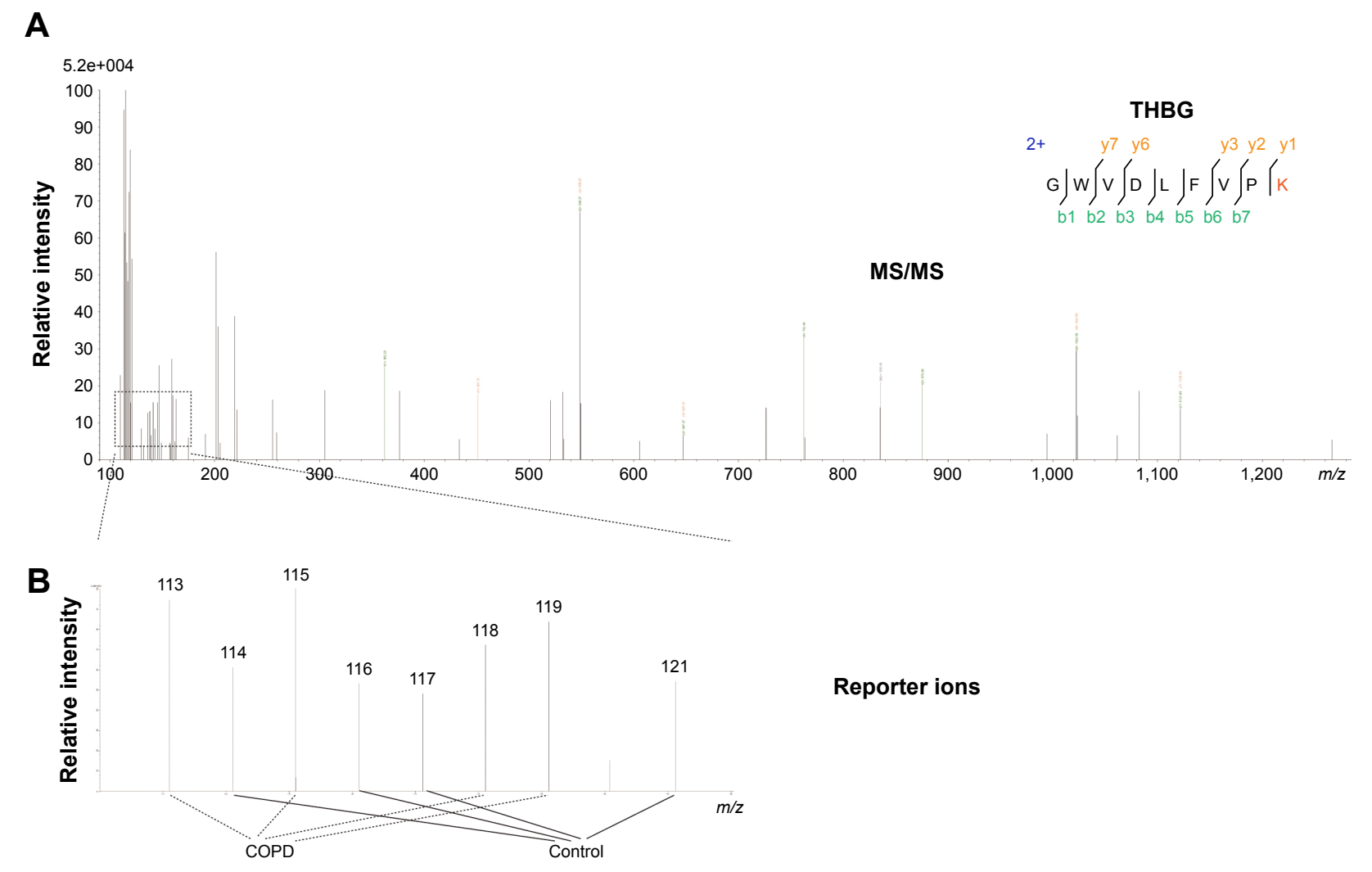

Figure 2 Identification of THBG by iTRAQ.

Notes: (A) Representative MS/MS (-GWVDLFVPK-) spectra of THBG; 5 typical $y$-ions and 7 b-ions were identified by iTRAQ, as presented in the upper panel. (B) Four COPD samples were labeled by reporter ions $113,115,118$, and II9; 4 controls were labeled by reporter ions 114 , II6, II7, and I2I. Relative intensity represents the relative abundance of THBG in the samples.

Abbreviations: COPD, chronic obstructive pulmonary disease; iTRAQ, isobaric tags for relative and absolute quantification; MS/MS, tandem mass spectrometry; THBG, thyroxine-binding globulin. 
Table I Baseline data of subjects in the verification panel

\begin{tabular}{|c|c|c|c|c|c|c|}
\hline \multirow[t]{2}{*}{ Variables } & \multicolumn{2}{|c|}{ COPD vs control } & \multicolumn{4}{|c|}{ GOLD I-IV vs control } \\
\hline & $\begin{array}{l}\text { Control } \\
(n=33)\end{array}$ & $\begin{array}{l}\text { COPD } \\
(n=53)\end{array}$ & $\begin{array}{l}\text { GOLD I } \\
(n=10)\end{array}$ & $\begin{array}{l}\text { GOLD II } \\
(n=15)\end{array}$ & $\begin{array}{l}\text { GOLD III } \\
(n=19)\end{array}$ & $\begin{array}{l}\text { GOLD IV } \\
(n=9)\end{array}$ \\
\hline Age, years & $58 \pm 5.1$ & $64 \pm 5.4 * *$ & $66 \pm 3.4 * *$ & $63 \pm 6.5 * *$ & $64 \pm 5.5^{* *}$ & $62 \pm 4.4 * *$ \\
\hline $\mathrm{BMI}, \mathrm{kg} / \mathrm{m}^{2}$ & $24 \pm 3.1$ & $24 \pm 3.5$ & $25 \pm 2.2$ & $25 \pm 3.6$ & $24 \pm 3.7$ & $22 \pm 3.9$ \\
\hline Smoking history, pack-years & $31 \pm 7.2$ & $38 \pm 10 * *$ & $39 \pm 3.1^{*}$ & $38 \pm 10 *$ & $38 \pm 14^{*}$ & $39 \pm 5.5^{*}$ \\
\hline Smoking cessation duration, years & $10 \pm 2.4$ & $11 \pm 3.1$ & $11 \pm 3.4$ & $13 \pm 3.1 * *$ & $10 \pm 3.0$ & $9.4 \pm 1.9$ \\
\hline $\mathrm{FEV}_{1}$, \%pred & $94 \pm 13$ & $52 \pm 21 * *$ & $84 \pm 2.9$ & $64 \pm 9.6 * *$ & $40 \pm 5.5^{* *}$ & $24 \pm 4.2^{* *}$ \\
\hline RV, \%pred & $90 \pm 36$ & $157 \pm 75^{* *}$ & $86 \pm 60$ & $137 \pm 59 * *$ & $162 \pm 45^{* *}$ & $259 \pm 53 * *$ \\
\hline RV/TLC, \% & $28 \pm 10$ & $52 \pm 16 * *$ & $35 \pm 13$ & $46 \pm 15^{* *}$ & $56 \pm 8.2 * *$ & $70 \pm 7.7^{* *}$ \\
\hline LAA, \% ${ }^{\mathrm{a}}$ & $2.1 \pm 1.4$ & $12 \pm 10 * *$ & $5.5 \pm 3.4$ & $6.0 \pm 4.7$ & $15 \pm 10 * *$ & $23 \pm 12 * *$ \\
\hline $\mathrm{AE}^{\mathrm{b}}$ & None & $16(30 \%)$ & 0 & $6(40 \%)$ & $6(32 \%)$ & $4(44 \%)$ \\
\hline Diabetes mellitus & I (3.0\%) & $3(5.7 \%)$ & $2(20 \%)$ & 0 & 0 & I (II\%) \\
\hline Hypertension & $2(6.1 \%)$ & $12(23 \%)^{*}$ & $2(20 \%)$ & $4(27 \%)$ & $4(2 \mid \%)$ & $2(22 \%)$ \\
\hline Abnormal TT3, higher/lower & 0 & 0 & 0 & 0 & 0 & 0 \\
\hline Abnormal FT3, higher/lowerc & I (3.0\%) & I (I.9\%) & 0 & 0 & I (5.3\%) & 0 \\
\hline Abnormal TT4, higher/lower ${ }^{c}$ & I (3.9\%) & $2(3.8 \%)$ & 0 & I (6.3\%) & $\mathrm{I}(5.3 \%)$ & I (II\%) \\
\hline Abnormal FT4, higher/lower ${ }^{c}$ & 0 & 0 & 0 & 0 & 0 & 0 \\
\hline Abnormal TSH, higher/lower ${ }^{c}$ & 0 & I (I.9\%) & I (I0\%) & 0 & 0 & 0 \\
\hline THBG, $\mu g / m L$ & $6.6 \pm 2.0$ & $9.2 \pm 2.3 * *$ & $7.3 \pm 1.9$ & $9.4 \pm 2.0 * *$ & $8.8 \pm 1.6 * *$ & $12 \pm 2.3 * *$ \\
\hline Peroxirodoxin-2, ng/mL & $44 \pm 9.1$ & $43 \pm 34$ & $39 \pm 14$ & $52 \pm 61$ & $37 \pm 14$ & $43 \pm 10$ \\
\hline Galectin-7, pg/mL & $567 \pm 606$ & $303 \pm 274$ & $552 \pm 435$ & $155 \pm 125$ & $243 \pm 196$ & $337 \pm 290$ \\
\hline AIAT, mg/mL & $5.4 \pm 0.49$ & $5.4 \pm 0.67$ & $5.7 \pm 0.53$ & $5.3 \pm 0.91$ & $5.4 \pm 0.60$ & $5.4 \pm 0.59$ \\
\hline Fibrinogen, g/L & $2.7 \pm 0.7$ & $3.3 \pm 0.8 * *$ & $3.1 \pm 0.54$ & $3.3 \pm 1.0$ & $3.5 \pm 0.66 * *$ & $3.6 \pm 1.0 * *$ \\
\hline
\end{tabular}

Notes: Continuous variables are shown as mean \pm SD; categorical variables were presented as number $(\%)$. $* P<0.05$ or $* * P<0.01:$ COPD patients were compared with controls. aAA, \%: the extent of the low-attenuation area assessed by computed tomography. ${ }^{b} \mathrm{AE}$ : the number of subjects with $\mathrm{AE}$ in a $\mathrm{I}$-year follow-up period. 'Abnormal: the indexes are not within the following normal range: TT3 (0.6-I.8I ng/mL); FT3 (2.3-4.2 pg/mL); TT4 (4.5-10.9 $\mu \mathrm{g} / \mathrm{mL})$; FT4 (0.89-I.8 ng/dL); TSH (0.55-4.78 $\mu$ IU/mL).

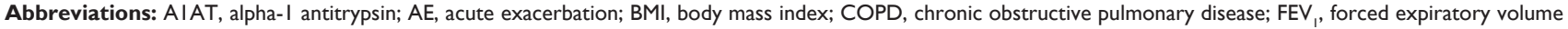
in I s; GOLD, Global Initiative for Obstructive Lung Disease; LAA, low-attenuation area; pred, predicted; RV, residual volume; TLC, total lung capacity; SD, standard deviation; THBG, thyroxine-binding globulin; TT3, total T3; FT3, free T3; TT4, total T4; FT4, free T4; TSH, thyroid stimulating hormone.

Age, smoking history, hypertension, and smoking cessation duration were significantly different between COPD patients and controls, as well as between subgroups of COPD patients and controls, and they needed to be corrected by multivariate linear regression model and covariance analyses as potential confounders. Galectin-7 and peroxiredoxin-2 were not significantly different between groups, as shown in Figure S3.

Levels of THBG were higher in COPD patients (9.2 \pm $2.3 \mu \mathrm{g} / \mathrm{mL})$ than in controls $(6.6 \pm 2.0 \mu \mathrm{g} / \mathrm{mL})$. ANOVA with a post hoc test indicated that THBG levels in GOLD stages II $(9.4 \pm 2.0 \mu \mathrm{g} / \mathrm{mL})$, III $(8.8 \pm 1.6 \mu \mathrm{g} / \mathrm{mL})$, and IV $(12 \pm 2.3 \mu \mathrm{g} / \mathrm{mL})$ patients were elevated, but no shift was observed in GOLD I patients $(7.3 \pm 1.9 \mu \mathrm{g} / \mathrm{mL})$, compared with controls $(6.6 \pm 2.0 \mu \mathrm{g} / \mathrm{mL})$. Fibrinogen, a relatively acknowledged marker, was selected as a reference. Fibrinogen levels were significantly different among the different groups. However, a post hoc test indicated that fibrinogen levels in GOLD III and IV patients were only slightly elevated compared with those in controls. The corresponding bar chart is presented in Figure 3. Covariance analysis and a multivariate linear regression model, correcting for age, smoking history, hypertension, and smoking cessation duration, demonstrated that the group (COPD vs control; or GOLD I-IV vs control) was still an independent factor that influenced THBG levels, as shown in Table S3.

Alpha-1 antitrypsin (A1AT), also called serpin family A member 1 (SERPINA1), plays a key role in the pathogenesis of emphysema. THBG, also called serpin family A member 7 (SERPINA7), belongs to serpin family A. Thus, the levels of A1AT were detected as a reference. However, the levels of A1AT were not significantly different among different groups. More details are shown in Figure S4.

\section{Monitoring values of THBG for the progression of COPD}

To explore whether THBG could assist in the management of COPD, ROC curves for THBG, fibrinogen, and their combination were generated and are presented in Figure 4. Figure 4A indicates that both THBG (AUC: $0.814 ; 95 \%$ confidence interval [CI]: 0.716-0.890) and fibrinogen (AUC: 0.739 ; 95\% CI: $0.633-0.828$ ) were slightly able to differentiate between COPD patients and controls. Figure 4B indicates that THBG (AUC: 0.786; 95\% CI: 0.652-0.887) 
A

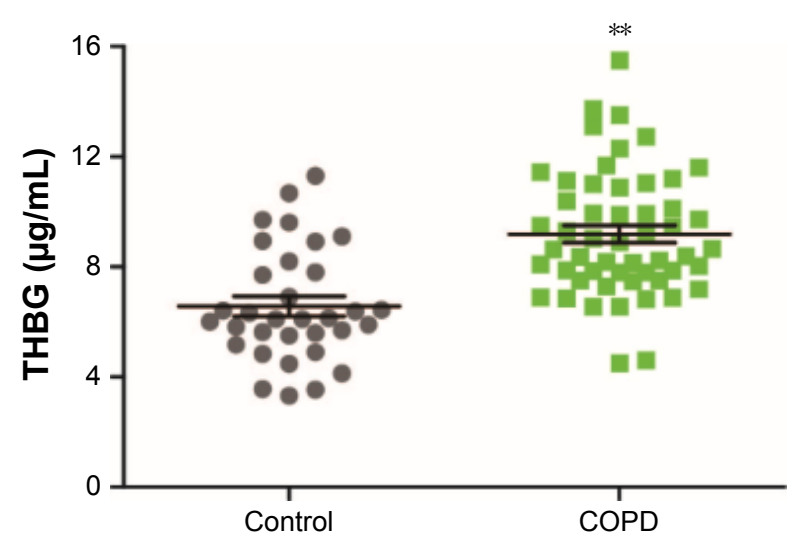

C

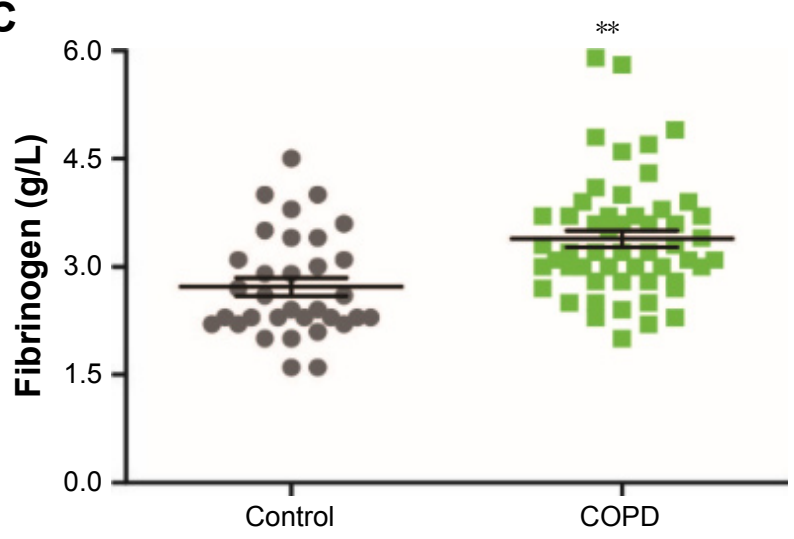

B

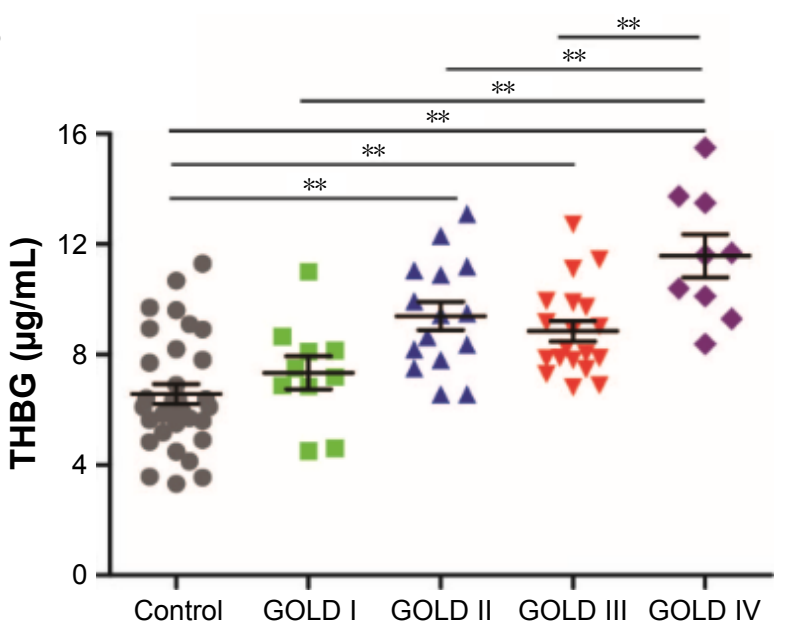

D

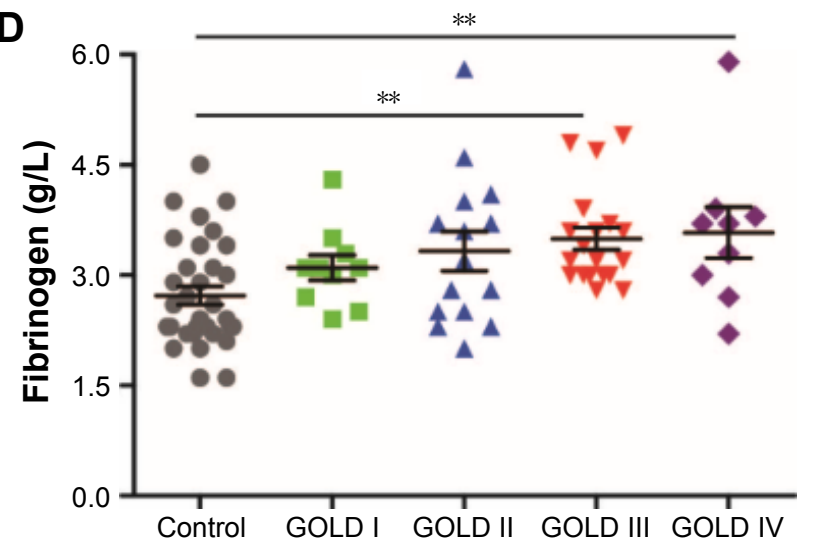

Figure 3 Levels of THBG and fibrinogen measured by ELISA in the verification panel.

Notes: (A) and (B) represent two different grouping types of THBG (control vs COPD and control vs GOLD I-IV, respectively); (C) and (D) represent two different grouping types of fibrinogen, respectively. Control: $n=33$ smokers without COPD; COPD: $n=53$ smokers with COPD ( $=10$ GOLD I; $n=15$ GOLD II; $n=19$ GOLD III; $n=9$ GOLD IV). Mean values ( \pm SE) are indicated with a horizontal line and error bar. $* * P<0.01$.

Abbreviations: COPD, chronic obstructive pulmonary disease; ELISA, enzyme-linked immunosorbent assay; GOLD, Global Initiative for Obstructive Lung Disease; $\mathrm{SE}$, standard error; THBG, thyroxine-binding globulin.

could distinguish between GOLD I and GOLD II-IV patients, whereas fibrinogen (AUC: 0.623; 95\% CI: $0.480-0.753$ ) could not. Figure $4 \mathrm{C}$ indicates that THBG (AUC: $0.639 ; 95 \% \mathrm{CI}: 0.495-0.766$ ) and fibrinogen (AUC: 0.616 ; 95\% CI: 0.472-0.746) could not distinguish between GOLD I, II and GOLD III, IV patients. Figure 4D indicates that THBG (AUC: 0.851; 95\% CI: 0.726-0.934) was better able to distinguish between GOLD I-III and GOLD IV patients than could fibrinogen (AUC: $0.582 ; 95 \%$ CI: 0.439-0.716). The combination of THBG and fibrinogen outperformed the separate factors in Figure 4A-C. Cutoff values with the corresponding sensitivity and specificity are described in Table S4.

\section{Correlation of THBG with lung function}

THBG was negatively associated with $\mathrm{FEV}_{1} \%$ pred $(r=-0.559, P=0.000)$ and positively associated with RV/ TLC $(r=0.464, P=0.000)$, RV\%pred $(r=0.381, P=0.000)$, and LAA\% $(r=0.446, P=0.000)$, as shown in Figure $5 \mathrm{~A}-\mathrm{D}$, respectively. Due to the association of age with THBG ( $r=0.246, P=0.022$ ), age was recognized as a confounder. More details are listed in Table S5.

The partial correlation and multivariate linear regression model, corrected for age, indicated that THBG was still associated with $\mathrm{FEV}_{1}$ \%pred, RV/TLC\%, RV\%pred, and LAA\%, as shown in Table S6.

\section{Relationship between baseline THBG levels and $A E$}

In the 1-year follow-up program, AE information was collected from COPD patients. The COPD patients were divided into high- and low-level groups based on the median level of THBG $(8.66 \mu \mathrm{g} / \mathrm{mL})$. The Kaplan-Meier curve with log-rank test $(P=0.014)$ showed that the occurrence time of AEs in the high-level group was shorter than that in the low-level group, as presented in Figure 6. 
A

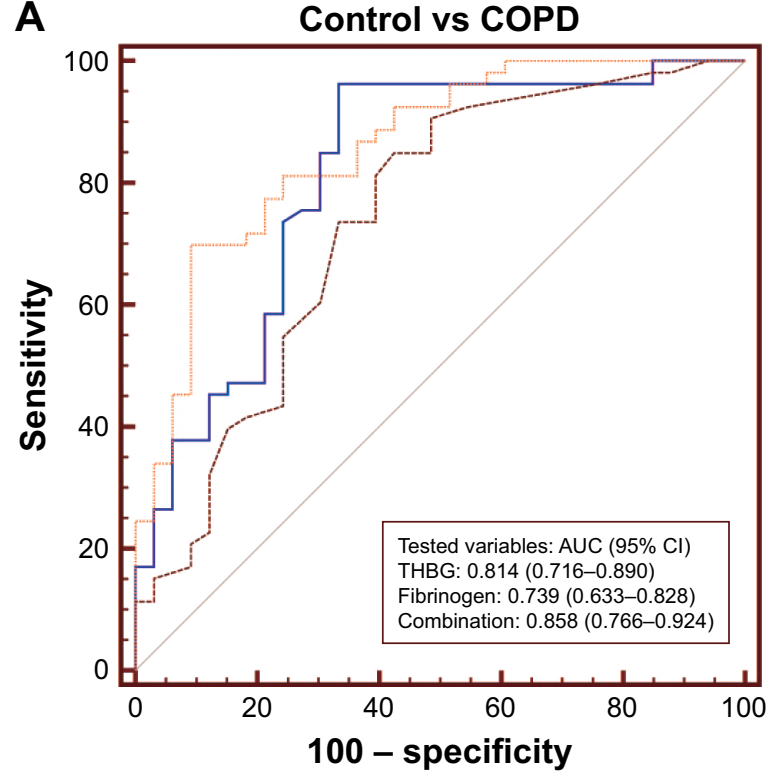

C

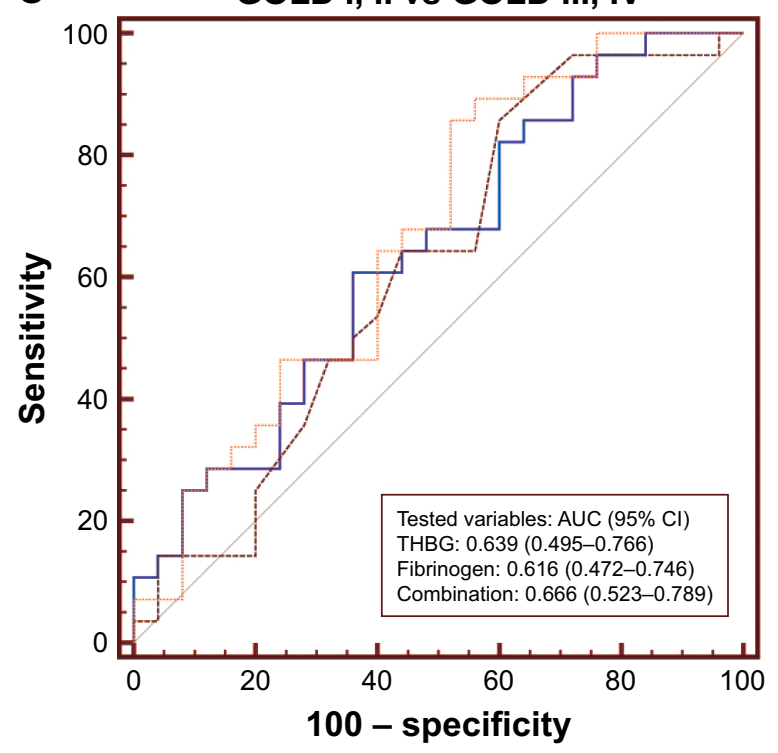

B GOLD I vs GOLD II-IV

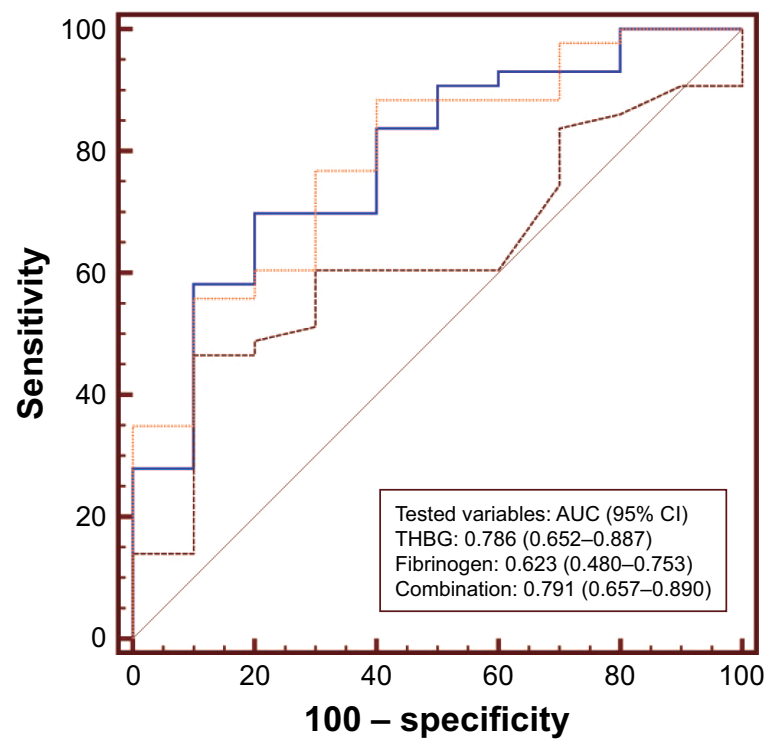

D GOLD I-III vs GOLD IV

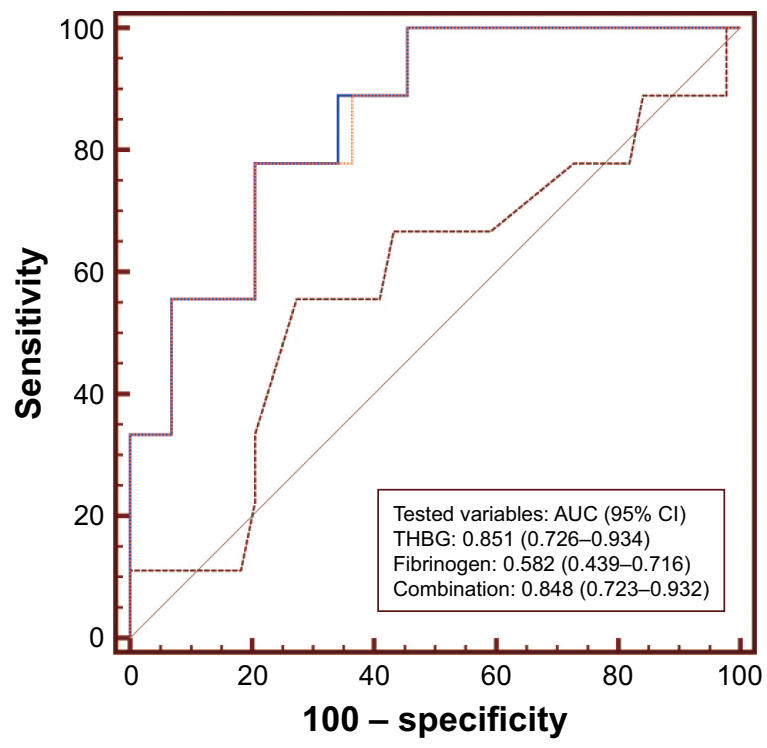

Diagonal — THBG -..-... Fibrinogen $\cdots$

Figure 4 Diagnostic value of THBG, fibrinogen, and their combination for COPD in the verification panel.

Notes: ROC curves were derived to compare the AUCs of THBG, fibrinogen, and their combination between (A) COPD patients of GOLD stages I-IV and controls without COPD; (B) COPD patients of GOLD I stage and COPD patients of GOLD II-IV stages; (C) COPD patients of GOLD I and II stages and COPD patients of GOLD III and IV stages; (D) COPD patients of GOLD I-III stages and COPD patients with GOLD IV. The greater AUCs mean greater diagnostic values.

Abbreviations: AUC, area under the curve; $\mathrm{Cl}$, confidence interval; COPD, chronic obstructive pulmonary disease; GOLD, Global Initiative for Obstructive Lung Disease; ROC, receiver operating characteristic; THBG, thyroxine-binding globulin.

Potential risk factors related to AE (ie, THBG level, $\mathrm{FEV}_{1} \%$ pred, age, smoking history, smoking cessation duration, hypertension, and diabetes mellitus) were analyzed using the Cox proportional hazards model. The results revealed that THBG level was an independent risk factor (HR: 4.229, $P=0.032$ ), as shown in Table S7. This finding indicated that AE risk in the high-level THBG group increased 3.229-fold compared with that in the low-level group.
Baseline THBG levels did not predict the alterations of $\mathrm{FEV}_{1} \%$ pred, $\mathrm{RV} / \mathrm{TLC} \%$, or $\mathrm{RV} \%$ pred within a 1 -year follow-up, as shown in Figure S5.

\section{Discussion}

THBG was identified by iTRAQ-labeling proteomics, and its plasma levels in COPD were elevated compared with those in controls and were associated with the severity of 

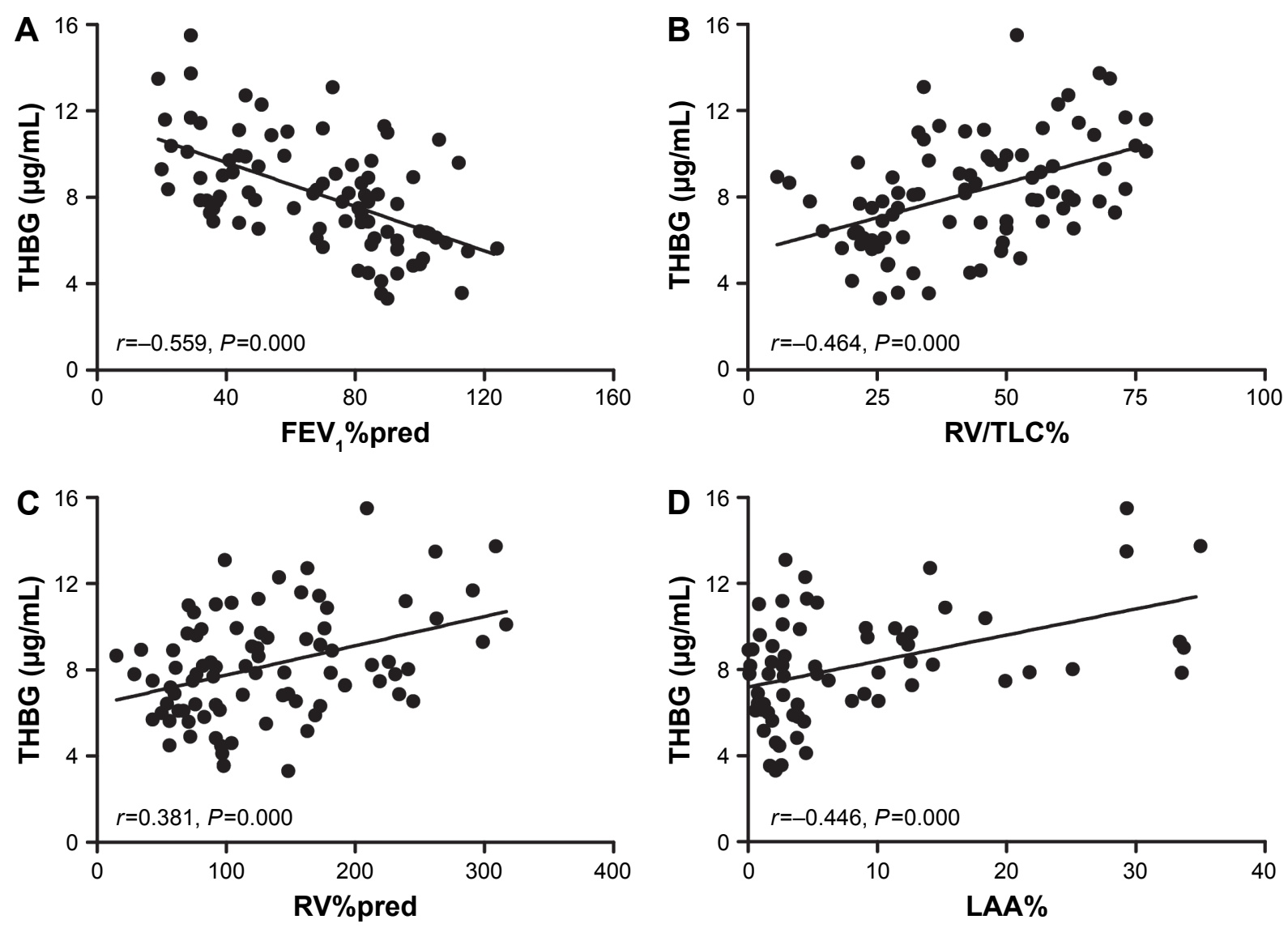

Figure 5 Correlations between THBG and baseline data in the verification panel.

Note: THBG was negatively correlated with (A) FEV \%pred and was positively correlated with (B) RV/TLC, (C) RV\%pred, and (D) LAA\%.

Abbreviations: FEV , forced expiratory volume in I s; RV, residual volume; LAA, low-attenuation area; pred, predicted; THBG, thyroxine-binding globulin; TLC, total lung capacity.

lung function. THBG was able to distinguish patients with worse lung function and performed better if combined with fibrinogen. Higher baseline THBG levels predicted a greater risk of AE.

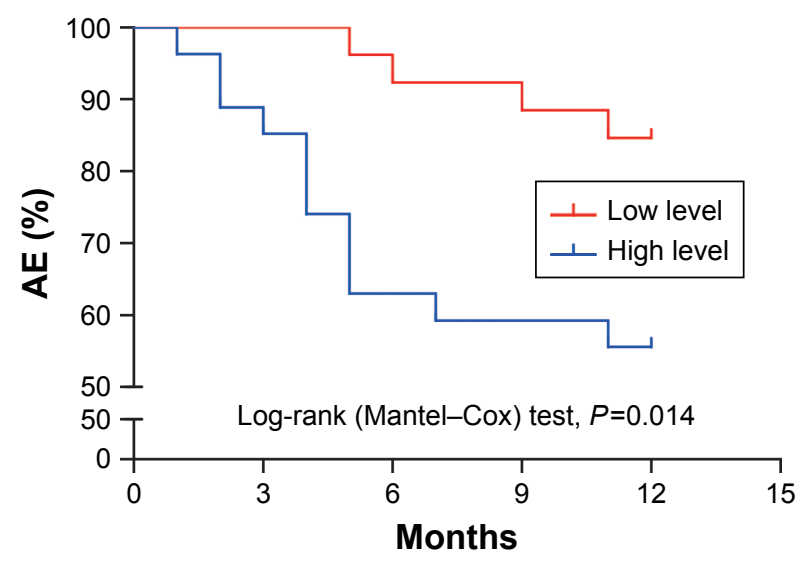

Figure 6 Kaplan-Meier curve for the first AE based on baseline THBG level. Note: A Kaplan-Meier curve was generated to compare the differences in the occurrence time of AEs between the low- and high-level THBG groups. Abbreviations: $A E$, acute exacerbation; THBG, thyroxine-binding globulin.
In total, 41 differentially expressed proteins were identified by proteomics in the discovery panel, some of which have been reported previously, such as platelet factor 4 and lipopolysaccharide-binding protein. ${ }^{30,31}$ These results suggested that iTRAQ-labeling proteomics has the potential to identify the markers of COPD. Global repeatability and quantification of the proteomic technique exhibited excellent performance, indicating that its identification and quantification abilities were reliable. The data verified by ELISA further demonstrated the reliability of proteomics.

Eventually, THBG, a hepatic-origin plasma protein, was identified and verified in the verification panel. The biological function of THBG is to carry thyroid hormones, ${ }^{32}$ and it binds $>70 \%$ of T3 and T 4 in circulation. ${ }^{33}$ Recently, researchers have emphasized a disorder of the endocrine system in patients with COPD. ${ }^{34,35}$ Several studies have found that subclinical hypothyroidism (with elevated TSH but without elevated T3 and T4) is related to frequent AEs and reduced lung function in COPD patients. ${ }^{34,35}$ In the study, no differences in thyroid function, including TSH, were observed between COPD 
patients and controls. However, THBG was elevated in COPD patients and was associated with AEs and the severity of lung function. Thus, the assumption is that increased THBG is also a feedback for "subclinical hypothyroidism" in COPD patients and even reflects an earlier alteration in these people. Unfortunately, THBG was not detected as a test item in these past studies. ${ }^{34,35}$ To further explore the relationship between COPD and thyroid function, it is recommended that THBG be measured in future studies. In addition to the well-known physiological function, structural features of THBG also draw our attention. A1AT (SERPINA1) is a key protease inhibitor involved in the progression of COPD with emphysema. ${ }^{36}$ In terms of genomics, THBG (SERPINA7) is also a serine protease inhibitor and may play an important role in the progression of COPD or emphysema. ${ }^{37}$

The value of monitoring THBG was evaluated using ROC curves. The study found that THBG levels in COPD patients were elevated compared with those in controls, and ROCs also suggested that THBG could differentiate COPD patients from controls, indicating that THBG is a potential marker for COPD. Although the independent diagnostic value of THBG and fibrinogen for COPD was not strong enough, their combination showed a larger clinical value. Thus, the combined application of multiple markers would be helpful for the management of COPD. Notably, THBG had a greater ability to identify patients with more severe lung function than fibrinogen, which offers a new strategy to monitor the progression of COPD patients.

The biological value of THBG was assessed by correlation analyses. The study revealed that THBG was associated with $\mathrm{FEV}_{1}$ \%pred, indicating that it is likely to be involved in the limitation of the airway; THBG was also related to $\mathrm{RV} \%$ pred, RV/TLC, and LAA\%, indicating that it possibly participates in the pathological process of emphysema. Moreover, the saturability of THBG is barely $>25 \%$ due to the low abundance of T3 and T4 in the bloodstream. ${ }^{38}$ Therefore, high levels of free THBG offer a basis for its involvement in other biological processes. In this study, A1AT (SERPINA1) was not altered, which was in accordance with previous studies among Chinese patients, ${ }^{39}$ but THBG (SERPINA7) was elevated in COPD patients. Therefore, it was considered worthwhile to further explore the potential clinical and biological value of THBG in patients with COPD.

The predictive capacity of baseline THBG for AEs was analyzed in a 1-year follow-up program. The Kaplan-Meier curve indicated that AEs occurred more often in patients with higher THBG levels than in those with lower THBG levels over a period of 1 year. The Cox proportional hazards model, corrected for $\mathrm{FEV}_{1}$ \%pred, age, smoking history, smoking cessation duration, hypertension, and diabetes mellitus, demonstrated that the baseline THBG level was still an independent factor that could predict the occurrence of AEs. Moreover, it also revealed that COPD patients with higher baseline THBG levels had greater risks of AEs than those with lower THBG levels. In addition, the relationship between baseline THBG and the progression of lung function was also explored. However, baseline THBG did not predict the minor alterations in lung function because of the shorter follow-up period in the study.

There are several merits in this study. 1) Another larger panel was selected to verify THBG levels rather than only the discovery panel. 2) The verification panel was followed up for a year, which allowed us to explore the relationship between THBG and AE. 3) A multivariate regression model, corrected for unmatched variables or risk factors, ensured the reliability of the conclusions.

There are several limitations in the study. 1) Only 8 participants were enrolled in the discovery panel because iTRAQ-labeling proteomics included only 8 different isotopic tags. 2) Only 3 candidate proteins identified by proteomics were verified by ELISA in the verification panel due to the limited availability of kits. 3) The population in this study was relatively low, which may have caused potential bias. Thus, the validity of THBG still needs to be verified in larger clinical trials in the future. 4) This pilot study found an association between THBG and lung function but did not reveal their causal relationship. In the future, the potential mechanism of THBG action in COPD will be our main study direction. 5) The relationship of THBG with treatment was not investigated in this study, which will be explored in future studies. 6) Nonsmokers without COPD were not included in this study, limiting our understanding of the relationships among THBG, smoking, and COPD. Nonsmokers should be included in future studies. 7) The lung function of subjects did not clearly change in the 1-year follow-up period, restricting further analyses of the progression of disease.

\section{Conclusion}

THBG is a potential plasma biomarker for COPD, which could assist us in the management of stable stages and AEs in COPD patients. It offers new insights into the pathogenesis of COPD.

\section{Acknowledgments}

The authors thank all contributors to the study. In addition, they thank BGI (Shenzhen, China), for performing the 
proteomic analyses. This work was supported by the National Natural Science Foundation of China (grant numbers 81270097 and 81470235) and Beijing Medical University (grant number 20110176). The funders had no role in study design, data collection and analysis, the decision to publish, or the preparation of the manuscript.

\section{Author contributions}

All authors declare their individual contributions to the article. Bei He completed the global design of the study and revised the article; Wenqi Diao conducted statistical analyses and interpretation and wrote the manuscript; Ning Shen and Yipeng Du enrolled subjects and obtained plasma samples; Xiaoyan Sun performed lung function tests for all subjects; Beibei Liu collected the clinical information of subjects; Ming $\mathrm{Xu}$ assisted in the revision of the manuscript. All authors contributed to the data analysis, drafting, and critical revision of the paper; approved the final version of the manuscript; and agreed to be accountable for all aspects of the work.

\section{Disclosure}

The authors report no conflicts of interest in this work.

\section{References}

1. Vestbo J, Hurd SS, Agusti AG, et al. Global strategy for the diagnosis, management, and prevention of chronic obstructive pulmonary disease: GOLD executive summary. Am J Respir Crit Care Med. 2013;187(4): 347-365.

2. Vafaee F. Using multi-objective optimization to identify dynamical network biomarkers as early-warning signals of complex diseases. Sci Rep. 2016;6:22023.

3. Lee KY, Chuang HC, Chen TT, et al. Proteoglycan 4 is a diagnostic biomarker for COPD. Int J Chron Obstruct Pulmon Dis. 2015;10: 1999-2007.

4. Narute $\mathrm{P}$, Seam N, Tropea M, et al. Temporal changes in microRNA expression in blood leukocytes from patients with the acute respiratory distress syndrome. Shock. Epub 2016 Nov 21.

5. Klupczynska A, Derezinski P, Garrett TJ, et al. Study of early stage non-small-cell lung cancer using Orbitrap-based global serum metabolomics. J Cancer Res Clin Oncol. 2017;143(4):649-659.

6. Vestbo J, Rennard S. Chronic obstructive pulmonary disease biomarkers for disease activity needed - urgently. Am J Respir Crit Care Med. 2010;182(7):863-864.

7. Koutsokera A, Kostikas K, Nicod LP, Fitting JW. Pulmonary biomarkers in COPD exacerbations: a systematic review. Respir Res. 2013; 14:111.

8. Thyagarajan B, Jacobs DR, Apostol GG, Smith LJ, Lewis CE, Williams OD. Plasma fibrinogen and lung function: the CARDIA Study. Int J Epidemiol. 2006;35(4):1001-1008.

9. Carter RI, Ungurs MJ, Pillai A, Mumford RA, Stockley RA. The relationship of the fibrinogen cleavage biomarker $\mathrm{A} \alpha-\mathrm{Val} 360$ with disease severity and activity in $\alpha 1$-antitrypsin deficiency. Chest. 2015;148(2): 382-388.

10. Janssen DJ, Mullerova H, Agusti A, et al; Eclipse Investigators. Persistent systemic inflammation and symptoms of depression among patients with COPD in the ECLIPSE cohort. Respir Med. 2014;108(11): $1647-1654$.
11. Agusti A, Edwards LD, Rennard SI, et al; Evaluation of COPD Longitudinally to Identify Predictive Surrogate Endpoints (ECLIPSE) Investigators. Persistent systemic inflammation is associated with poor clinical outcomes in COPD: a novel phenotype. PLoS One. 2012; 7(5): 37483.

12. Lock-Johansson S, Vestbo J, Sorensen GL. Surfactant protein D, club cell protein 16 , pulmonary and activation-regulated chemokine, $\mathrm{C}$-reactive protein, and fibrinogen biomarker variation in chronic obstructive lung disease. Respir Res. 2014;15:147.

13. Fang JY, Wang PW, Huang CH, Chen MH, Wu YR, Pan TL. Skin aging caused by intrinsic or extrinsic processes characterized with functional proteomics. Proteomics. 2016;16(20):2718-2731.

14. Poersch A, Grassi ML, Carvalho VP, et al. A proteomic signature of ovarian cancer tumor fluid identified by high throughput and verified by targeted proteomics. J Proteomics. 2016;145:226-236.

15. Zhang Y, Chen X, Hu Y, et al. Preliminary characterizations of a serum biomarker for sarcoidosis by comparative proteomic approach with tandem-mass spectrometry in ethnic Han Chinese patients. Respir Res. 2013;14:18.

16. O'Neil SE, Sitkauskiene B, Babusyte A, et al. Network analysis of quantitative proteomics on asthmatic bronchi: effects of inhaled glucocorticoid treatment. Respir Res. 2011;12:124.

17. Ishikawa N, Ohlmeier S, Salmenkivi K, et al. Hemoglobin alpha and beta are ubiquitous in the human lung, decline in idiopathic pulmonary fibrosis but not in COPD. Respir Res. 2010;11:123.

18. Diao WQ, Shen N, Du YP, et al. Fetuin-B (FETUB): a plasma biomarker candidate related to the severity of lung function in COPD. Sci Rep. 2016;6:30045.

19. Lee EJ, In KH, Kim JH, et al. Proteomic analysis in lung tissue of smokers and COPD patients. Chest. 2009;135(2):344-352.

20. Auffray C, Adcock IM, Chung KF, Djukanovic R, Pison C, Sterk PJ. An integrative systems biology approach to understanding pulmonary diseases. Chest. 2010;137(6):1410-1416.

21. Gray RD, MacGregor G, Noble D, et al. Sputum proteomics in inflammatory and suppurative respiratory diseases. Am J Respir Crit Care Med. 2008;178(5):444-452.

22. Bozinovski S, Hutchinson A, Thompson M, et al. Serum amyloid-a is a biomarker of acute exacerbations of chronic obstructive pulmonary disease. Am J Respir Crit Care Med. 2008;177(3):269-278.

23. Chhabra SK, Gupta M. Exhaled breath condensate analysis in chronic obstructive pulmonary disease. Indian J Chest Dis Allied Sci. 2012;54(1):27-37.

24. Tu C, Mammen MJ, Li J, et al. Large-scale, ion-current-based proteomics investigation of bronchoalveolar lavage fluid in chronic obstructive pulmonary disease patients. J Proteome Res. 2014;13(2):627-639.

25. Terracciano R, Pelaia G, Preiano M, Savino R. Asthma and COPD proteomics: current approaches and future directions. Proteomics Clin Appl. 2015;9(1-2):203-220.

26. Wiktorowicz JE, Jamaluddin M. Proteomic analysis of the asthmatic airway. Adv Exp Med Biol. 2014;795:221-232.

27. Govender P, Dunn MJ, Donnelly SC. Proteomics and the lung: analysis of bronchoalveolar lavage fluid. Proteomics Clin Appl. 2009;3(9): 1044-1051.

28. Madani A, Van Muylem A, de Maertelaer V, Zanen J, Gevenois PA. Pulmonary emphysema: size distribution of emphysematous spaces on multidetector CT images - comparison with macroscopic and microscopic morphometry. Radiology. 2008;248(3):1036-1041.

29. Hubl W, Meissner D, Demant T, et al. Evaluation of the LIAISON thyroid chemiluminescence immunoassays. Clin Lab. 2000;46(3-4): 181-189.

30. Wedzicha JA, Syndercombe-Court D, Tan KC. Increased platelet aggregate formation in patients with chronic airflow obstruction and hypoxaemia. Thorax. 1991;46(7):504-507.

31. Groenewegen KH, Postma DS, Hop WC, et al. Increased systemic inflammation is a risk factor for COPD exacerbations. Chest. 2008;133(2): $350-357$. 
32. Gerhard DS, Wagner L, Feingold EA, et al; MGC Project Team. The status, quality, and expansion of the NIH full-length cDNA project: the Mammalian Gene Collection (MGC). Genome Res. 2004;14(10B): 2121-2127.

33. Refetoff S. Abnormal thyroid hormone transport. In: De Groot LJ, Beck-Peccoz P, Chrousos G, et al, editors. Endotext [Internet]. South Dartmouth, MA: MDText.com, Inc.; 2000. Available from: https:// www.ncbi.nlm.nih.gov/books/NBK285563/

34. SarincUlasli S, Bozbas SS, Ozen ZE, Ozyurek BA, Ulubay G. Effect of thyroid function on COPD exacerbation frequency: a preliminary study. Multidiscip Respir Med. 2013;8(1):64.

35. Terzano C, Romani S, Paone G, Conti V, Oriolo F. COPD and thyroid dysfunctions. Lung. 2014;192(1):103-109.
36. Morse JO. Alphal-antitrypsin deficiency (first of two parts). $N$ Engl J Med. 1978;299(19):1045-1048.

37. Hayashi Y, Mori Y, Janssen OE, et al. Human thyroxine-binding globulin gene: complete sequence and transcriptional regulation. Mol Endocrinol. 1993;7(8):1049-1060.

38. Wada N, Chiba H, Shimizu C, Kijima H, Kubo M, Koike T. A novel missense mutation in codon 218 of the albumin gene in a distinct phenotype of familial dysalbuminemic hyperthyroxinemia in a Japanese kindred. J Clin Endocrinol Metab. 1997;82(10):3246-3250.

39. Kwok JS, Lawton JW, Yew WW, Chau CH, Lee J, Wong PC. Protease inhibitor phenotypes and serum alpha-1-antitrypsin levels in patients with COPD: a study from Hong Kong. Respirology. 2004;9(2): 265-270. 


\section{Supplementary materials}

Table SI Baseline data of subjects in the discovery panel

\begin{tabular}{|c|c|c|}
\hline Variables & Control $(n=4)$ & COPD $(n=4)$ \\
\hline Age, years & $62 \pm 4$ & $63 \pm 5$ \\
\hline $\mathrm{BMI}, \mathrm{kg} / \mathrm{m}^{2}$ & $25 \pm 2$ & $22 \pm 4$ \\
\hline Smoking history, pack-years & $36 \pm 3$ & $37 \pm 5$ \\
\hline Smoking cessation duration, years & $7 \pm 3$ & $9 \pm 3$ \\
\hline $\mathrm{FEV}_{1}, \%$ pred & $82 \pm 17$ & $27 \pm 2$ \\
\hline RV, \%pred & $63 \pm 14$ & $273 \pm 50$ \\
\hline RV/TLC, \% & $29 \pm 6$ & $67 \pm 12$ \\
\hline LAA $\%$ & $0.94 \pm 0.92$ & $21 \pm 14$ \\
\hline Thyroxine-binding globulin, $\mu g / \mathrm{mL}$ & $6.6 \pm 1.1$ & $11 \pm 2$ \\
\hline Peroxirodoxin-2, ng/mL & $36 \pm 7$ & $28 \pm 5$ \\
\hline Galectin-7, pg/mL & $126 \pm 34$ & || $4 \pm 5 \mid$ \\
\hline
\end{tabular}

Abbreviations: BMI, body mass index; COPD, chronic obstructive pulmonary disease; FEV , forced expiratory volume in I s; LAA, low-attenuation area; pred, predicted; $\mathrm{RV}$, residual volume; TLC, total lung capacity.
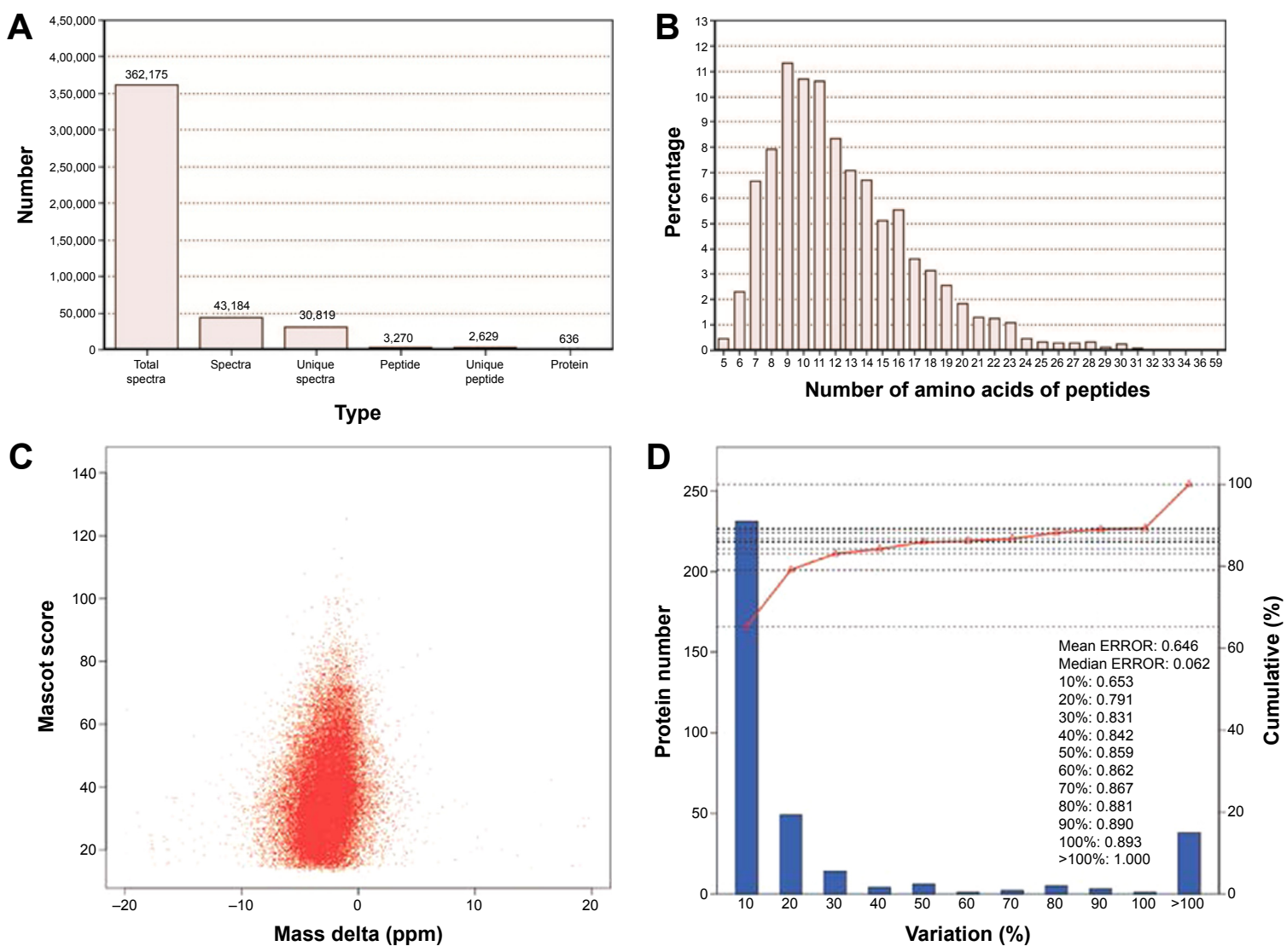

Figure SI Quality control of iTRAQ-labeling proteomics in the discovery panel.

Notes: (A) A total of 362,175 total spectra, 43,184 spectra and 30,819 unique spectra were identified, demonstrating that the number and quality of spectras used to identify proteins were enough. The number of peptides and unique peptides were 3,270 and 2,629, respectively, indicating that the number and quality of peptides used to quantify levels of proteins were enough. A total of 636 proteins were identified, suggesting that enough proteins were used to find the differentially expressed proteins. (B) Numbers of amino acids of most peptides identified by proteomics were distributed between 7 and 30 , indicating that the length of most peptides is appropriate. (C) Mass delta of most peptides were distributed within $10 \mathrm{ppm}(0.01 \mathrm{Da})$, indicating accuracy of identified peptides was good. The unique peptides, whose mascot scores were greater than 40 , were used to quantify levels of proteins in this study. (D) CV\% of $79.1 \%$ of proteins were less than $20 \%$, indicating that variation of quantifying results of protein levels were very small. The left $y$-axis reflects the blue bar graph and the right $y$-axis reflects the red line graph.

Abbreviations: COPD, chronic obstructive pulmonary disease; iTRAQ, isobaric tags for relative and absolute quantification; CV, coefficient of variation. 
Table S2 Differentially expressed proteins* between smokers with or without COPD

\begin{tabular}{|c|c|c|c|}
\hline Accession $^{\mathrm{a}}$ & Abbreviation & Description & Change fold \\
\hline \multicolumn{4}{|l|}{ Upregulated } \\
\hline B3KRN4 & HTRAI & Serine protease HTRAI & 1.28 \\
\hline P00739 & HPTR & Haptoglobin-related protein & 1.43 \\
\hline Q9UBXI & CATF & Cathepsin F & 1.24 \\
\hline PI9652 & AIAG2 & Alpha-I-acid glycoprotein 2 & 1.59 \\
\hline Q9UL89 & MIHCVR & Myosin-reactive immunoglobulin heavy chain variable region & 2.31 \\
\hline A0A087×0P6 & IGKV2D-29 & Protein IGKV2D-29 & 3.39 \\
\hline B3KS79 & AIAC & Alpha-I-antichymotrypsin & 1.39 \\
\hline P05543 & THBG & Thyroxine-binding globulin & 1.61 \\
\hline $\mathrm{P} 05160$ & $\mathrm{FI} 3 \mathrm{~B}$ & Coagulation factor XIII B chain & 1.23 \\
\hline P02776 & PLF4 & Platelet factor 4 & 1.82 \\
\hline AOAOAOMSI4 & IGHVI-45 & Protein IGHVI-45 & 2.31 \\
\hline Q5UGI6 & SERPINGI & Serine/cysteine proteinase inhibitor clade G member I splice variant 2 & 1.29 \\
\hline S6C4Q7 & IGHC & IgG H chain & 3.05 \\
\hline \multicolumn{4}{|c|}{ Downregulated } \\
\hline P27482 & CALL3 & Calmodulin-like protein 3 & 0.57 \\
\hline $\mathrm{P} 23142$ & FBLNI & Fibulin-I & 0.80 \\
\hline $\mathrm{PI} 8428$ & LBP & Lipopolysaccharide-binding protein & 0.81 \\
\hline A2MYD4 & A2MYD4 & V2-7 protein (fragment) & 0.71 \\
\hline Q53RT3 & APRVI & Retroviral-like aspartic protease I & 0.47 \\
\hline P0I608 & KVII6 & Ig kappa chain V-I region Roy & 0.61 \\
\hline P47929 & Gal-7 & Galectin-7 & 0.42 \\
\hline A0A075B730 & EPPKI & Epiplakin & 0.73 \\
\hline P0I6II & KVII9 & Ig kappa chain V-I region Wes & 0.50 \\
\hline Q15084 & PDIA6 & Protein disulfide-isomerase $\mathrm{A} 6$ & 0.57 \\
\hline G3V2WI & SERPINAIO & Protein Z-dependent protease inhibitor & 0.74 \\
\hline B3KRF9 & LP-54 & Leucine-rich repeat-containing protein 54 & 0.81 \\
\hline H0Y7V6 & PSPB & Pulmonary surfactant-associated protein B & 0.76 \\
\hline Q7Z35I & DKFZ & Putative uncharacterized protein DKFZp686N02209 & 0.49 \\
\hline P3II5I & SIOA7 & Protein SI00-A7 & 0.43 \\
\hline G3V4U0 & $\mathrm{F}-5$ & Fibulin-5 & 0.73 \\
\hline Q6U2E7 & $\mathrm{C} 4 \mathrm{BI}$ & C4BI (fragment) & 0.75 \\
\hline $\mathrm{A} 8 \mathrm{~K} 6 \mathrm{Cl}$ & CETP & Cholesteryl ester transfer protein, plasma & 0.71 \\
\hline P55072 & TERA & Transitional endoplasmic reticulum ATPase & 0.80 \\
\hline A0A087WXL8 & IGHG3 & Ig gamma- 3 chain $C$ region & 0.83 \\
\hline B4DF70 & Per-2 & Peroxiredoxin-2 & 0.64 \\
\hline B4DEW5 & Mul-2 & Multimerin-2 & 0.82 \\
\hline B4DTD5 & FA & Filamin-A & 0.57 \\
\hline P06727 & APOA4 & Apolipoprotein A-IV & 0.81 \\
\hline P0I78I & HV320 & Ig heavy chain $\mathrm{V}$-III region GAL & 0.78 \\
\hline P07237 & PDIAI & Protein disulfide-isomerase & 0.77 \\
\hline Q5FWF9 & IGL & IGL@ protein & 0.83 \\
\hline B5BU38 & Ann & Annexin & 0.65 \\
\hline
\end{tabular}

Notes: *Differentially expressed proteins: the change fold is $>1.2$ and $P$-value is $<0.05$ by Student's $t$-test. ${ }^{2}$ The accession of differentially expressed proteins is identified based on UniProt database.

Abbreviation: COPD, chronic obstructive pulmonary disease.

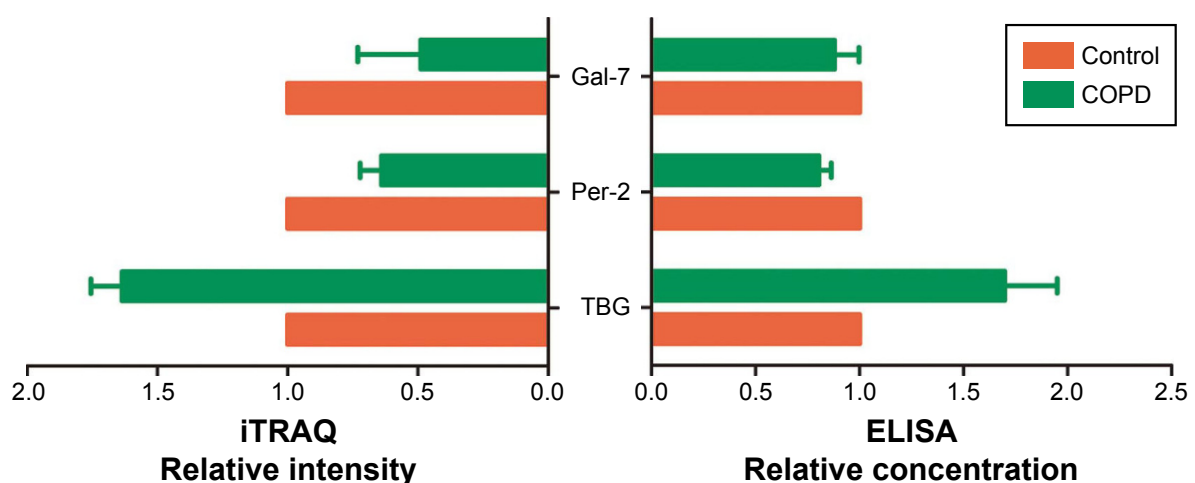

Figure S2 Verification for potential candidates using ELISA in the discovery panel.

Notes: To present the changing trend directly, controls of each group were adjusted to I. Relative intensity quantified by iTRAQ is presented in the left panel. Relative concentration quantified by ELISA is shown in the right panel. The results measured by iTRAQ and ELISA were similar.

Abbreviations: COPD, chronic obstructive pulmonary disease; ELISA, enzyme-linked immunosorbent assay; Gal-7, galectin-7; iTRAQ, isobaric tags for relative and absolute quantification; Per-2, peroxirodoxin-2; THBG, thyroxine-binding globulin. 

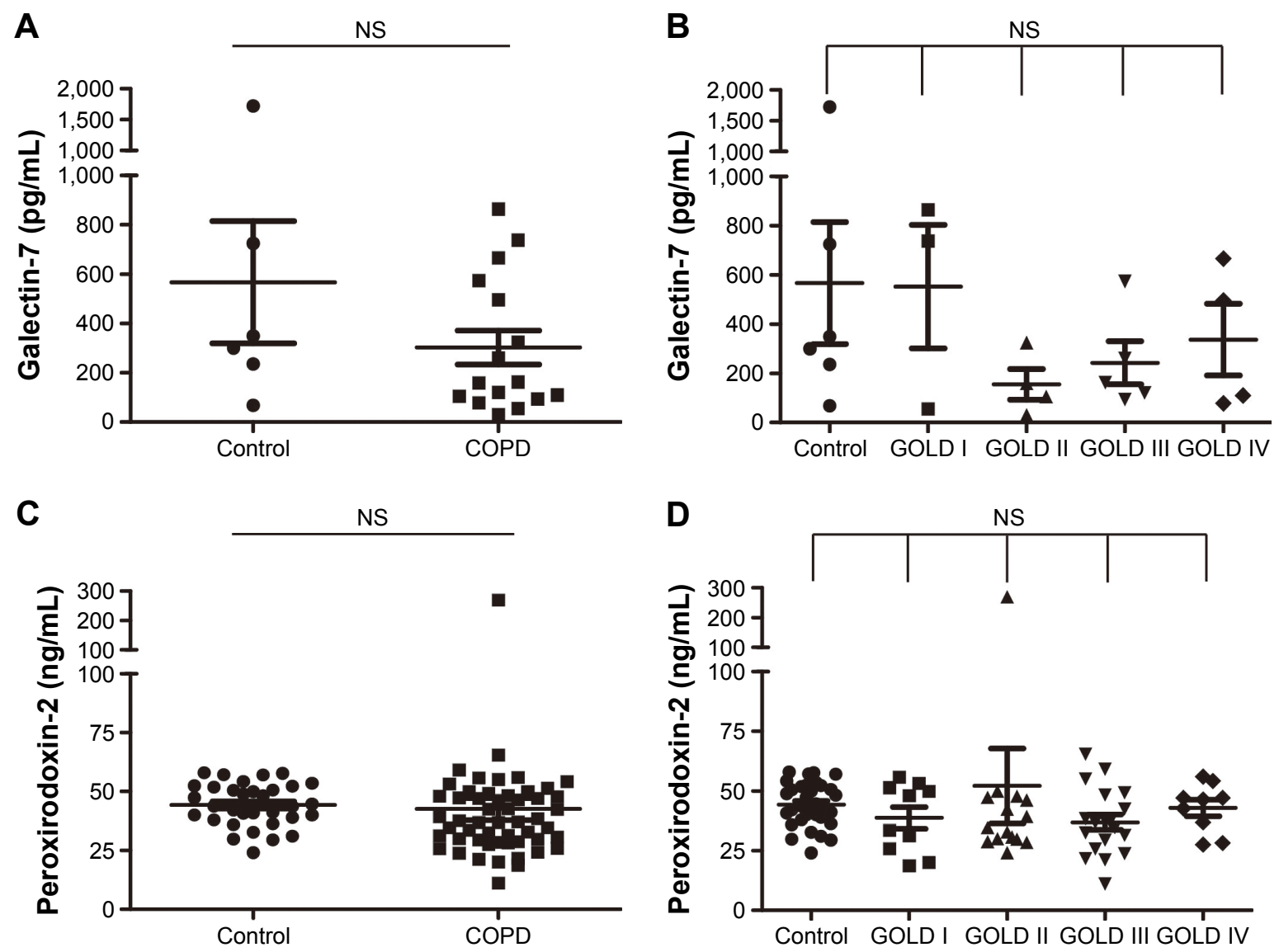

Figure S3 Levels of galectin-7 and peroxirodoxin-2 between groups in the verification panel.

Notes: $(\mathbf{A})$ and $(\mathbf{B})$ represent two different grouping types of galectin-7 (control vs COPD and control vs GOLD I-IV, respectively); (C) and (D) represent two different grouping types of peroxirodoxin-2 (control vs COPD and control vs GOLD I-IV, respectively). Due to plasma levels of galectin-7 being very low, it was only detected in 22 subjects ( $n=6$, control; $n=3$, GOLD I; $n=4$, GOLD II; $n=5$, GOLD III; and $n=4$, GOLD IV). Galectin-7 and peroxirodoxin-2 in plasma were not statistically different among different groups.

Abbreviations: COPD, chronic obstructive pulmonary disease; GOLD, Global Initiative for Obstructive Lung Disease; NS, not significant.

Table S3 Corrected difference of THBG levels between groups by multivariate models

\begin{tabular}{lll}
\hline Variables & Covariance analysis & Multivariate linear regression \\
\hline COPD vs control & & $P=0.000$ \\
Group (COPD vs control) & $P=0.000$ & $P=0.969$ \\
Age, years & $P=0.969$ & $P=0.264$ \\
Smoking history, pack-years & $P=0.264$ & $P=0.957$ \\
Hypertension & $P=0.957$ & $P=0.000$ \\
GOLD I-IV vs control & & $P=0.621$ \\
Group (GOLD I-IV vs control) & $P=0.000$ & $P=0.227$ \\
Age, years & $P=0.536$ & $P=0.402$ \\
Smoking history, pack-years & $P=0.21$ I & $P=0.620$ \\
Smoking cessation duration, years & &
\end{tabular}

Notes: Covariance analysis and multivariate linear regression model, which were corrected for age, smoking history, and hypertension between COPD and controls, indicated that the group (COPD vs control) is the unique variable influencing THBG levels. Similarly, multivariate models, which were corrected for age, smoking history, and smoking cessation duration among controls, GOLD I-IV, implied that the group (GOLD I-IV vs control) is the independent factor against THBG levels.

Abbreviations: COPD, chronic obstructive pulmonary disease; GOLD, Global Initiative for Obstructive Lung Disease; THBG, thyroxine-binding globulin. 
A

NS

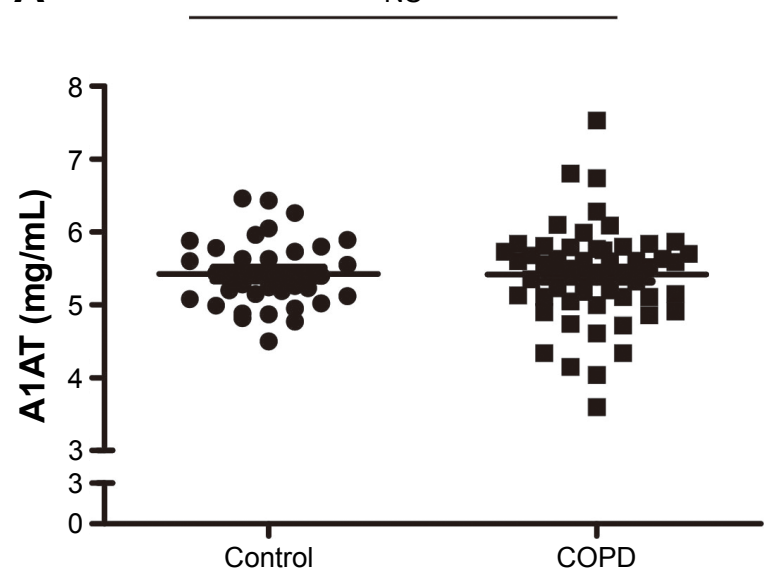

B

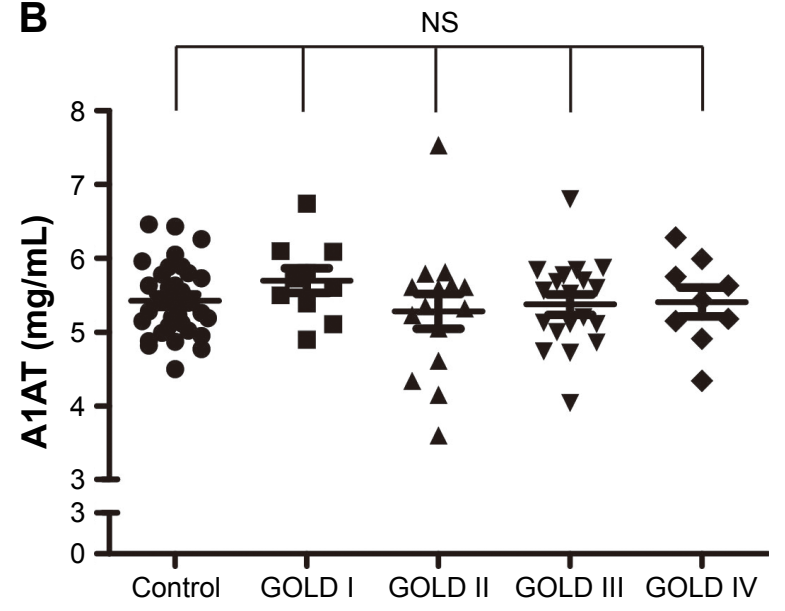

Figure $\mathbf{S} 4$ Levels of AIAT between groups in the verification panel.

Notes: (A) Compared levels of AIAT between control and COPD; and (B) compared levels between control and GOLD I-IV. AIAT (SERPINAI) and THBG (SERPINA7) are serpin family A (SERPINA)-I and -7, respectively; thus, the levels of AIAT were also detected as a reference. Levels of AIAT were not significantly different among different groups.

Abbreviations: AIAT alpha-I antitrypsin; COPD, chronic obstructive pulmonary disease; GOLD, Global Initiative for Obstructive Lung Disease; NS, not significant; THBG, thyroxine-binding globulin.

Table S4 Area under the curve and cutoff values of THBG, fibrinogen, and their combination

\begin{tabular}{|c|c|c|c|c|c|c|}
\hline \multirow[t]{2}{*}{ Comparison } & \multicolumn{3}{|c|}{ Area under the curve ( $95 \%$ confidence interval) } & \multicolumn{3}{|c|}{ Cutoff value (sensitivity, specificity) } \\
\hline & THBG & Fibrinogen & Combination & THBG & Fibrinogen & Combination \\
\hline COPD vs & 0.814 & 0.739 & 0.858 & $6.42 \mu \mathrm{g} / \mathrm{mL}$ & $2.603 \mathrm{~g} / \mathrm{L}$ & 0.682 \\
\hline control & $(0.716-0.890)$ & $(0.633-0.828)$ & $(0.766-0.924)$ & $(96.2 \%, 66.7 \%)$ & $(84.9 \%, 57.6 \%)$ & $(69.8 \%, 90.9 \%)$ \\
\hline GOLD I vs & 0.786 & 0.623 & 0.791 & $8.14 \mu g / \mathrm{mL}$ & $3.5 \mathrm{~g} / \mathrm{L}$ & 0.6463 \\
\hline GOLD II-IV & $(0.652-0.887)$ & $(0.480-0.753)$ & $(0.657-0.890)$ & $(69.8 \%, 80.0 \%)$ & $(46.5 \%, 90.0 \%)$ & $(88.4 \%, 60.0 \%)$ \\
\hline GOLD I, II vs & 0.639 & 0.616 & 0.666 & $8.66 \mu \mathrm{g} / \mathrm{mL}$ & $2.8 \mathrm{~g} / \mathrm{L}$ & 0.435 \\
\hline GOLD III, IV & $(0.495-0.766)$ & $(0.472-0.746)$ & $(0.523-0.789)$ & $(60.7 \%, 64 \%)$ & $(85.7 \%, 40.0 \%)$ & $(85.7 \%, 48.0 \%)$ \\
\hline GOLD I-III vs & 0.851 & 0.582 & 0.848 & $9.94 \mu \mathrm{g} / \mathrm{mL}$ & $3.6 \mathrm{~g} / \mathrm{L}$ & 0.154 \\
\hline GOLD IV & $(0.726-0.934)$ & $(0.439-0.716)$ & $(0.723-0.932)$ & $(77.8 \%, 79.5 \%)$ & $(55.6 \%, 72.7 \%)$ & $(77.8 \%, 79.5 \%)$ \\
\hline
\end{tabular}

Abbreviations: COPD, chronic obstructive pulmonary disease; GOLD, Global Initiative for Obstructive Lung Disease; THBG, thyroxine-binding globulin.

Table S5 The association of THBG and clinical indexes of subjects

\begin{tabular}{lll}
\hline Variables & Univariate correlation \\
\hline Pearson correlation & & $P=0.022^{*}$ \\
Age, years & $r=0.246$ & $P=0.662$ \\
BMl, kg/m & $r=-0.048$ & $P=0.453$ \\
Smoking history, pack-years & $r=0.082$ & $P=0.426$ \\
Smoking cessation duration, years & $r=0.087$ & $P=0.000^{* *}$ \\
FEV, \%pred & $r=-0.559$ & $P=0.000^{* *}$ \\
RV/TLC, \%pred & $r=0.464$ & $P=0.000^{* *}$ \\
RV, \%pred & $r=0.381$ & $P=0.000^{* *}$ \\
LAA, \% & $r=0.446$ & \\
Spearman correlation & & $P=0.171$ \\
Hypertension & $r=-0.149$ & $P=0.45 I$ \\
Diabetes mellitus & $r=-0.082$ & $N o n e$ \\
Abnormal TT3 & None & $P=0.755$ \\
Abnormal FT3 & $r=0.034$ & $P=0.163$ \\
Abnormal TT4 & $r=0.152$ & None \\
Abnormal FT4 & None & $P=0.536$ \\
Abnormal TSH & $r=-0.068$ & \\
\hline
\end{tabular}

Notes: $* P<0.05$ or $* * P<0.0$ I: the variable was correlated with THBG levels. Pearson coefficient reflected the association of continuous variables with THBG, and Spearman coefficient reflected the association of categorical variables with THBG.

Abbreviations: BMI, body mass index; FEV , forced expiratory volume in I s; LAA, low-attenuation area; RV, residual volume; THBG, thyroxine-binding globulin; TLC, total lung capacity; TT3, total T3; FT3, free T3; TT4, total T4; FT4, free T4; TSH, thyroid-stimulating hormone; pred, predicted. 
Table S6 Corrected association of THBG with main clinical indexes by multivariate models

\begin{tabular}{|c|c|c|c|c|}
\hline \multirow{2}{*}{$\begin{array}{l}\text { Variables } \\
\mathrm{FEV}_{1} \text {, \%pred }\end{array}$} & \multicolumn{2}{|c|}{ Partial correlation } & \multicolumn{2}{|c|}{ Multivariate linear regression } \\
\hline & $r^{\prime}=-0.523$ & $P=0.000$ & $\beta^{\prime}=-0.535$ & $P=0.000$ \\
\hline RV/TLC, \%pred & $r^{\prime}=0.407$ & $P=0.000$ & $\beta^{\prime}=0.446$ & $P=0.000$ \\
\hline RV, \%pred & $r^{\prime}=0.348$ & $P=0.001$ & $\beta^{\prime}=0.345$ & $P=0.001$ \\
\hline LAA, \% & $r^{\prime}=0.4 \mid 4$ & $P=0.000$ & $\beta^{\prime}=0.427$ & $P=0.000$ \\
\hline
\end{tabular}

Note: Partial correlation and multivariate linear regression models, corrected for age, suggested that THBG was still associated with FEV \%pred, RV/TLC\%, RV\%pred, and LAA\%.

Abbreviations: $\mathrm{FEV}_{1}$, forced expiratory volume in I s; LAA, low-attenuation area; pred, predicted; RV, residual volume; THBG, thyroxine-binding globulin; TLC, total lung capacity.

Table S7 HR of THBG for AE based on Cox proportional hazards models

\begin{tabular}{|c|c|c|}
\hline \multirow[t]{2}{*}{ Variables } & \multicolumn{2}{|c|}{ Cox proportional hazards models (enter method") } \\
\hline & HR $(95 \% \mathrm{Cl})$ & $P$-value \\
\hline THBG_level ${ }^{\mathrm{a}}$ & $4.229(1.13|-| 5.8 \mid 4)$ & 0.032 \\
\hline FEV, \%pred & $0.976(0.946-1.008)$ & 0.146 \\
\hline Age, years & $0.921(0.819-1.036)$ & 0.172 \\
\hline Smoking history, pack-years & $1.007(0.954-1.064)$ & 0.792 \\
\hline Smoking cessation duration, years & I.I37 (0.954-1.355) & 0.153 \\
\hline Hypertension & $0.668(0.177-2.525)$ & 0.552 \\
\hline Diabetes mellitus & $1.475(0.177-12.303)$ & 0.719 \\
\hline
\end{tabular}

Notes: ${ }^{2}$ THBG_level: It was divided into the high- and low-level groups based on the median of THBG $(8.66 \mu \mathrm{g} / \mathrm{mL})$. It was still an independent risk factor (HR: 4.229 , $P=0.032$ ) for $A E$, when THBG level, FEV \%pred, age, smoking history, smoking cessation duration, hypertension, and diabetes mellitus were corrected for. "Enter method means that all variables were included in Cox proportional hazards model.

Abbreviations: $\mathrm{AE}$, acute exacerbation; $\mathrm{Cl}$, confidence interval; $\mathrm{FEV}_{1}$, forced expiratory volume in I s; HR, hazard ratio; pred, predicted; THBG, thyroxine-binding globulin.

A

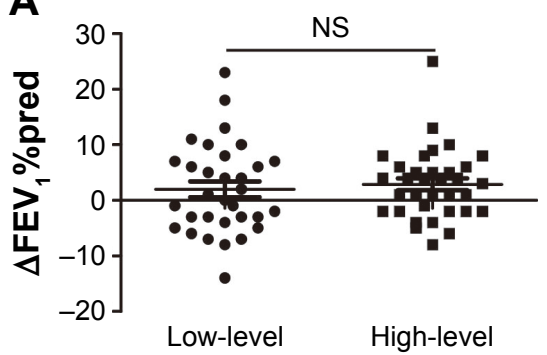

B

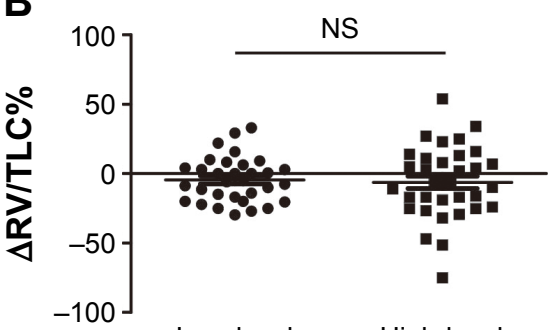

Low-level
C

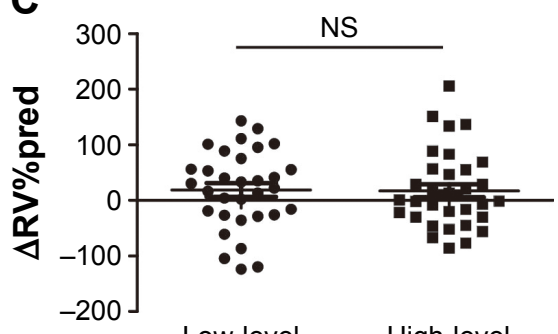

Low-level

High-level

Figure S5 Predicting the ability of baseline THBG for alteration of FEV \%pred, RV/TLC\%, and RV\%pred within I-year follow-up.

Notes: (A), (B) and (C) represent the difference between low- and high- level group in $\triangle \mathrm{FEV} \%$ pred, $\triangle \mathrm{RV} / \mathrm{TLC}$ and $\triangle \mathrm{RV} \%$ pred, respectively. $\Delta \mathrm{FEV}, \%$ pred represents $\mathrm{FEV}, \%$ pred before follow-up minus $\mathrm{FEV}$ \%pred after follow-up. Computing method of $\triangle \mathrm{RV} / \mathrm{TLC}$ and $\triangle \mathrm{RV} \%$ pred was the same as for FEV \%pred. Subjects were divided into the high- and low-level groups based on the median of THBG $(7.96 \mu \mathrm{g} / \mathrm{mL})$ in all subjects. Control: $n=29 ;$ COPD: $n=37$. Baseline THBG levels did not predict the progression of FEV , RV/TLC, and RV in this follow-up program.

Abbreviations: COPD, chronic obstructive pulmonary disease; $\mathrm{FEV}_{1}$, forced expiratory volume in I s; NS, not significant; pred, predicted; RV, residual volume; THBG, thyroxine-binding globulin; TLC, total lung capacity.

International Journal of COPD

\section{Publish your work in this journal}

The International Journal of COPD is an international, peer-reviewed journal of therapeutics and pharmacology focusing on concise rapid reporting of clinical studies and reviews in COPD. Special focus is given to the pathophysiological processes underlying the disease, intervention programs, patient focused education, and self management protocols.

\section{Dovepress}

This journal is indexed on PubMed Central, MedLine and CAS. The manuscript management system is completely online and includes a very quick and fair peer-review system, which is all easy to use. Visit $\mathrm{http}: / /$ www.dovepress.com/testimonials.php to read real quotes from published authors. 